\title{
Does My Patient with Multiple Comorbidities Have Heart Failure with Preserved Ejection Fraction, and Does It Matter?
}

Dmitry Abramov, $M D$

Heart Failure with Preserved Ejection Fraction (HFpEF) is a common diagnosis and accounts for half or more of all cases of heart failure. Despite its high prevalence and significant morbidity, the pathophysiology of HFpEF remains incompletely understood. Patients diagnosed with HFpEF often have significant cardiac and extra-cardiac comorbidities. Given the availability of evidence-based treatments for common comorbidities, but not for HFpEF, the necessity of diagnosing HFpEF among symptomatic elderly patients with multiple comorbidities is unclear. This commentary raises the question of whether the search for the diagnosis of $\mathrm{HFpEF}$ should instead be refocused to the management of common comorbidities without necessitating the heart failure diagnosis. (J Am Board Fam Med 2019;32:424-427.)

Keywords: Comorbidity, Heart Failure, Stroke Volume

Heart failure with preserved ejection fraction (HFpEF) comprises about half of the cases of heart failure in the United States. ${ }^{1}$ Despite its growing prevalence and significant morbidity, HFpEF remains incompletely understood and diagnosis in the community setting may be difficult. Widely used diagnostic criteria that incorporate clinical findings with laboratory values and echocardiographic parameters lack sensitivity which may result in underdiagnosis. $^{2}$ Overdiagnosis based on vague symptoms and nonspecific diagnostic parameters may likewise be problematic. The concern of over- and underdiagnosis is further compounded by the lack of evidence-based treatments for those diagnosed with HFpEF. Patients with HFpEF tend to be older, with multiple cardiac and noncardiac comordbitities. $^{3}$ This begs the question of whether diagnosis of $\mathrm{HFpEF}$ remains critical to optimize the care of

This article was externally peer reviewed.

Submitted 1 November 2018; revised 14 January 2019; accepted 15 January 2019.

From the Department of Cardiology, Loma Linda University, Loma Linda, CA.

Funding: none.

Conflict of interest: none declared.

Corresponding author: Dmitry Abramov, MD, Loma Linda University, 11234 Anderson St., Schuman Pavilion, SP1617, Loma Linda, CA 92354 (E-mail: Dabramov@llu.edu). predominantly older patients with multiple comorbidities.

\section{Defining Heart Failure with Preserved Ejection Fraction}

Heart failure has been defined as the inability of the heart to generate adequate cardiac output or the ability to generate adequate cardiac output only in the setting of elevated filling pressures. ${ }^{4}$ The standard clinical diagnostic criteria for HFpEF includes heart failure symptoms (such as dyspnea, orthopnea, paroxysmal nocturnal dyspnea) and elevated filling pressures (predominantly Wedge pressure) at rest or with exercise. ${ }^{5}$ Elevated filling pressures may be clinically estimated through measurement of jugular venous distention. Confirmation of elevated filling pressures, and therefore the heart failure diagnosis, can be obtained through invasive right heart catheterization. The history and physical examination-derived signs and symptoms suggestive of heart failure may be difficult to ascertain, and their correlation with invasively derived filling pressures has largely been based on studies of acutely decompensated or end-stage patients with reduced ejection fraction. ${ }^{1,6}$ Recently, permanently implanted pulmonary artery monitors have been used to help evaluate central filling pressures as a supplement to the physical examination, ${ }^{7}$ although 
the utility of such devices as part of the diagnostic evaluation of heart failure may be limited.

Current diagnostic criteria for HFpEF from the American College of Cardiology and American Heart Association include clinical signs or symptoms, ejection fraction $\geq 50 \%$, and evidence of diastolic dysfunction. ${ }^{1}$ Patients meeting heart failure criteria with ejection fraction of $41 \%$ to $49 \%$ can be classified as having heart failure with borderline or midrange ejection fraction, which is more clinically similar to HFpEF than to heart failure with reduced ejection fraction. ${ }^{1,8}$ Diagnostic criteria for HFpEF from the European Society of Cardiology are similar, and include signs and symptoms, ejection fraction of $\geq 50 \%$, elevated B-type natriuretic peptide values and echocardiographic evidence of structural abnormalities or diastolic dysfunction. ${ }^{9}$

\section{Limitations of Diagnostic Criteria for HFpEF}

While seemingly straightforward, the application of these criteria to the community setting is fraught with limitations. These diagnostic criteria have been criticized for low sensitivity, ${ }^{2}$ and the reliance on echocardiographic evaluation of diastolic dysfunction may neither accurately evaluate cardiac relaxation abnormalities nor contain sufficient sensitivity or specificity to detect a clinical course consistent with HFpEF. ${ }^{10}$ Although exact criteria for grading of diastolic dysfunction have changed over time,${ }^{11}$ diastolic abnormalities on echocardiography are common in community cohorts of patients with comorbidities such as obesity, hypertension, diabetes and coronary disease, ${ }^{12}$ and have poor correlation with heart failure symptoms. ${ }^{12,13}$ In addition, only minimal abnormalities in diastolic parameters (including a high prevalence of normal diastolic function, normal left atrial size, and normal wall thickness) are noted in cohorts with symptomatic HFpEF. ${ }^{10}$ Echocardiographic diastolic parameters may primarily estimate ventricular filling pressures, ${ }^{11}$ rather than necessarily providing an explanation for their elevation.

Clinical trials and day-to-day care of patients with HFpEF highlight the high burden of comorbidities associated with this condition. Common noncardiac comorbidities include older age, chronic kidney disease, and lung disease. Common cardiac comorbidities include diabetes, hypertension, coronary artery disease, obesity and atrial fibrillation. As dyspnea is a common symptom in patients with many of these comorbidities, and given the notable limitations of the diagnostic algorithms, how are clinicians to identify which of these patients may have HFpEF?

In light of these limitations, some have proposed scoring systems to help identify patients with HF$\mathrm{pEF}$ among those being evaluated for dyspnea ${ }^{5}$; identifying that the biggest predictors of elevated filling pressures (and therefore the HFpEF diagnosis) among patients with dyspnea are advanced age ( $>60$ years old), obesity (Body Mass Index $>30$ $\mathrm{kg} / \mathrm{m}^{2}$ ) and atrial fibrillation. Other factors having a smaller association with elevated filling pressures include elevated pulmonary artery systolic pressure, treatment with multiple antihypertensive medications, and abnormal diastolic parameter of E/e'. Having the 3 risk factors of advanced age, obesity, and atrial fibrillation was associated with a greater than $90 \%$ likelihood of meeting criteria for the diagnosis of HFpEF based on elevated filling pressures. ${ }^{5}$ Importantly, elevated B-type natriuretic peptide, dilated left atrium, ventricular hypertrophy, or abnormal ventricular strain-all key parameters associated with diastolic dysfunction-had either smaller or no independent predictive ability to diagnose elevated filling pressures.

This focus on comorbidities as the key contributor to pathophysiology of HFpEF raises significant questions about our current understanding of what it means to impart onto a patient the heart failure diagnosis. Dyspnea is a ubiquitous and subjective symptom in older individuals, ${ }^{14}$ and is particularly common in patients with comorbidities including obesity and atrial fibrillation. Dyspnea may also be multifactorial, and not primarily caused by elevation in intracardiac filling pressures. ${ }^{15} \mathrm{El}-$ evated filling pressures, even in the presence of dyspnea, may not always reflect heart failure, as every patient with end-stage renal disease on dialysis is not generally considered to have "heart failure" before a dialysis session.

\section{Refocus on Patient Management}

Instead of focusing on the diagnosis of $\mathrm{HFpEF}$, (ie, attempting to determine whether dyspnea is cardiac in origin or whether echocardiographic abnormalities are relevant to the clinical presentation), perhaps a better approach would be to return to the diagnosis and management of key cardiac and extracardiac comorbidities ${ }^{3}$ that are common in el- 
derly patients with limitations to functional capacity. It should be acceptable to diagnose and manage conditions associated with fluid retention, such as anemia, obesity, diabetes, kidney disease, or atrial fibrillation without invoking the heart failure diagnosis. Each of these comorbidities is a significant stand-alone diagnosis, associated with its own symptoms (often dyspnea), diagnostic criteria, and evidence-based treatments. ${ }^{3}$ Whether these comorbidities contribute to fluid retention through: their association with diastolic abnormalities, other ventricular myocardial abnormalities, their own unique pathophysiologies, or a combination of these remains unclear. Diuretics are the mainstay treatment of symptoms of volume overload regardless of etiology, and can be used to control symptoms regardless of whether the patient is diagnosed with HF$\mathrm{pEF}$, is suspected as having $\mathrm{HFpEF}$, or is presumed to have volume overload from a noncardiac etiology. Among patients with $\mathrm{HFpEF}$, the diuretic spironolactone is primarily effective at lower ejection fractions $(<50 \%)$, and the benefits among patients with higher ejection fractions are less certain. ${ }^{16}$

While the search for underlying pathophysiology continues, there is increasing evidence that comorbidities are the most prominent contributors to symptoms among patients labeled as having HFpEF. If symptomatic comorbidities require another name or label, perhaps a better one is "comorbidity associated heart failure" or even "comorbidity associated diuretic dependence." Employing terminology to more closely correlate with etiology based on subpopulations of HFpEF patients may simplify the diagnosis of a widely encountered clinical syndrome and help guide management in a way not currently possible with HFpEF.

May the diagnosis of HFpEF become more clinically relevant if ongoing trials of novel therapies, including sacubitril valsartan, empagliflozin, and others demonstrate improvement in clinical outcomes? The answer to that question has yet to be determined, as these medications may yield similar benefit in high risk primary prevention populations without the necessity of a HFpEF diagnosis. While diagnosing specific diseases that have previously fallen under the HFpEF umbrella such as Amyloid or Fabry's cardiomyopathy are increasingly critical as treatments become available, the benefit of diagnosing comorbidity associated $\mathrm{HFpEF}$ remains unclear. In addition, while diagnosing HFpEF may carry prognostic implications, the associated adverse prognosis may result more from a particularly advanced comorbidity burden rather than primary cardiac pathology or heart failure itself.

In conclusion, diagnosing HFpEF in the community may not be easy or even clinically relevant, and the answer to the question of, "does my patient with multiple comorbidities have HFpEF?" may be less important than using available treatments to improve the quality of life of symptomatic patients.

To see this article online, please go to: http://jabfm.org/content/ 32/3/424.full.

\section{References}

1. Yancy CW, Jessup M, Bozkurt B, et al. 2013 ACCF/AHA guideline for the management of heart failure: executive summary: A report of the American College of Cardiology Foundation/ American Heart Association Task Force on practice guidelines. Circulation 2013;128:1810-52.

2. Obokata M, Kane G, Reddy Y, Olson T, Melenovsky $\mathrm{V}$, Borlaug B. The role of diastolic stress testing in the evaluation for heart failure with preserved ejection fraction: An invasive-echocardiographic study. J Am Coll Cardiol 2017;69:879.

3. Shah SJ, Gheorghiade M. Heart failure with preserved ejection fraction: Treat now by treating comorbidities. JAMA 2008;300:431-3.

4. Denolin H, Kuhn H, Krayenbuehl HP, Loogen F, Reale A. The definition of heart failure. Eur Heart J 1983;4:445-8.

5. Reddy YNV, Carter RE, Obokata M, Redfield MM, Borlaug BA. a simple, evidence-based approach to help guide diagnosis of heart failure with preserved ejection fraction. Circulation 2018;138:861-70.

6. Thibodeau JT, Drazner MH. The role of the clinical examination in patients with heart failure. JACC Heart Fail 2018;6:543-51.

7. Adamson PB, Abraham WT, Bourge RC, et al. Wireless pulmonary artery pressure monitoring guides management to reduce decompensation in heart failure with preserved ejection fraction. Circ Heart Fail 2014;7:935-44.

8. Hsu JJ, Ziaeian B, Fonarow GC. Heart failure with mid-range (borderline) ejection fraction: Clinical implications and future directions. JACC Heart Fail 2017;5:763-71.

9. Ponikowski P, Voors AA, Anker SD, et al. 2016 ESC Guidelines for the diagnosis and treatment of acute and chronic heart failure: The Task Force for the diagnosis and treatment of acute and chronic heart failure of the European Society of Cardiology (ESC) Developed with the special contribution of the Heart Failure Association (HFA) of the ESC. Eur Heart J 2016;37:2129-200. 
10. Vaidya GN, Abramov D. Echocardiographic evaluation of diastolic function is of limited value in the diagnosis and management of HFpEF. J Card Fail 2018;24:392-6.

11. Nagueh SF, Smiseth OA, Appleton CP, et al. Recommendations for the evaluation of left ventricular diastolic function by echocardiography: An Update from the American Society of Echocardiography and the European Association of Cardiovascular Imaging. J Am Soc Echocardiogr 2016;29: $277-314$.

12. Redfield MM, Jacobsen SJ, Burnett JC, et al. Burden of systolic and diastolic ventricular dysfunction in the community. JAMA 2003;289:194-202.
13. Abhayaratna WP. Characteristics of Left Ventricular Diastolic Dysfunction In The Community: an Echocardiographic Survey. Heart 2006;92:1259-64.

14. van Mourik Y, Rutten FH, Moons KG, Bertens LC, Hoes AW, Reitsma JB. Prevalence and underlying causes of dyspnoea in older people: A systematic review. Age Ageing 2014;43:319-26.

15. Packer M. Questioning the obvious: does dyspnoea really matter in heart failure? Eur Heart J 2018;39: 2822-4.

16. Solomon SD, Claggett B, Lewis EF, et al. Influence of ejection fraction on outcomes and efficacy of spironolactone in patients with heart failure with preserved ejection fraction. Eur Heart J 2016;37:455-62. 